\title{
Protein Crosslinking Onto Gold Nanoparticles by the Use of Gamma Radiation
}

\section{Janaína A G Barros ${ }^{1 *}$, Jorge G S Batista ${ }^{1}$, Vitor de Moraes Zamarion ${ }^{2}$ and Ademar B Lugão ${ }^{1}$}

${ }^{1}$ Instituto de Pesquisas Energéticas e Nucleares (IPEN-CNEN/SP) - Avenida Lineu

Prestes, São Paulo, SP, Brazil

${ }^{2}$ Fundamental Chemistry Department, University of São Paulo (IQ-USP) Avenida

Lineu Prestes, São Paulo, SP, Brazil

*Corresponding Author: Janaína A G Barros, Instituto de Pesquisas Energéticas e

Nucleares (IPEN-CNEN/SP) - Avenida Lineu Prestes, São Paulo, SP, Brazil.
Received: November 30, 2021

Published: February 21, 2022

(C) All rights are reserved by Janaína A G

Barros., et al.

\begin{abstract}
Gold nanoparticles (AuNP) are being investigated for diagnostic and therapeutic nanomedicines considering their low toxicity and stability against oxidation, among other features. AuNP may be administered site-specifically or intravenously for diagnostic imaging by computed tomography or for therapy. Albumin functionalization of AuNP whether linked by chemical or physical processes may provide unique features to the system regarding biopharmaceutical aspects. Recently albumin crosslinking has been studied by radiation induced methods. This work aimed the development of radiation crosslinked bovine serum albumin (BSA) capped AuNP for improved administration of the nanoparticles. For such purpose AuNP were synthesized using 2.5 mM sodium tetrachloroaurate (III) dehydrate and $1.67 \mathrm{mM}$ resveratrol in water. The mixture was allowed to stand for 12 hours. AuNP capping was performed by BSA addition followed by slow addition of ice-cold ethanol to reach $5 \mathrm{mg} \mathrm{mL}^{-1} \mathrm{BSA}$ in a $30 \% \mathrm{v} / \mathrm{v}$ ) ethanol solution. The mixture was allowed to rest overnight at $\pm 4{ }^{\circ} \mathrm{C}$. BSA crosslinking onto AuNP was performed by gamma irradiation at doses of $2.5,5,7.5,10$ and $15 \mathrm{kGy}$. AuNP were synthesized with approximately $38 \mathrm{~nm}$, whereas BSA Capped AuNP presented size ranging from $70 \mathrm{~nm}$ up to $200 \mathrm{~nm}$, as revealed by dynamic light scattering. AuNP and BSA capped AuNP morphology was evaluated by transmission electron microscopy using negative staining and revealed protein aggregation onto AuNPs and its surroundings. Irradiation led to no shifts in maximum absorbance for both nanoparticles. Protein crosslinking was confirmed by bityrosine and increased with dose.
\end{abstract}

Keywords: Gold Nanoparticles; Bovine Serum Albumin; Resveratrol; Green Synthesis; BSA Capping; Functionalized Nanoparticles; Protein Crosslinking; Gamma Radiation

\section{Introduction}

Generally, nanoparticles are prepared by a variety of chemical and physical methods which are quite expensive and potentially hazardous to the environment which involve use of toxic and perilous chemicals that are responsible for various biological risks. The development of biologically-inspired experimental processes for the syntheses of nanoparticles is evolving into an important branch of nanotechnology.
An ecofriendly route of nanoparticle synthesis does not employ toxic chemicals, phytochemicals' use in diverse chemical transformation is well-known and the production of nanoparticles under nontoxic green conditions is important for medical and technological applications [1-3].

Gold nanoparticles (AuNP) are being investigated for diagnostic and therapeutic nanomedicines considering their low toxicity and stability against oxidation, among other features. AuNP may be ad- 
ministered site-specifically or intravenously for diagnostic imaging by computed tomography or for therapy $[4,5]$.

Resveratrol is known as a natural dietary phytochemical that has a preventive effect against breast cancer. Due to properties as antioxidant, anti-inflammatory, and antisenescence, and its potential as an anticancer drug is currently being evaluated in clinical trials for various types of cancers. Sanna., et al. noticed that the nanoformulation of phytochemicals shows enhanced cellular uptake, bioavailability, and anticancer activity as compared to phytochemicals [6-8].

Tipe., et al. [9] related the potential of resveratrol as reducing agents for the synthesis of gold nanoparticles, as well as on their putative effectiveness when attached on the gold nanoparticles surface. Tipe., et al. provided compelling evidence that the increased corona of resveratrol on AuNPs enhances the bioavailability of resveratrol so that therapeutically active species can be optimally available in vivo for applications in cancer therapy.

Albumin functionalization of AuNP, whether linked by chemical or physical processes, may provide unique features to the system regarding biopharmaceutical aspects [10]. Bolaños., et al. [11] compiled articles that demonstrate the synergy between serum albumin and gold nanoparticles. Capping AuNPs with albumin increase their colloidal stability and reduce their interaction with plasma proteins, besides albumin to favor the selective delivery of AuNPs. The system demonstrates significant potential in terms of spatial and temporally controlled release, in addition to its applications in theranostics, using simple detection techniques. Coating AuNPs with SA decreases the interaction of the nanosytem with endogenous proteins in the body, protecting the cargo against degradation.

There are many strategies for AuNP conjugation with albumin, resulting structures can be observed as AuNPs with a protein "halo" or take the form of nanocapsules in which AuNPs are confined in a cavity surrounded by albumin [11]. Initially albumin serum attaching to AuNPs through physical adsorption.

Serum albumin crosslinking has been studied by radiation induced methods [12,13]. Queiroz., et al. [12] demonstrated the synthesis of BSA nanoparticles using $\gamma$-irradiation at the dose of $10 \mathrm{kGy}$ in the presence of a cosolvent allowed the formation of the nanoparticles without the use of any chemical. The BSA crosslinked was revealed by the bityrosine formation monitored by fluorescence spectroscopy.

This work aimed the development of radiation crosslinked bovine serum albumin (BSA) capped AuNP for improved administration of the nanoparticles.

\section{Experimental}

\section{Materials}

Sodium tetrachloroaurate (III) dehydrate and Bovine Serum Albumin (BSA, Heat shock fraction, $\geq 98 \%$ purity) were purchased from Sigma-Aldrich ${ }^{\circledR}$ (USA). Resveratrol ( $\geq 98 \%$ purity) was acquired from Sunrise Chemical Co. (China). Ethanol, anhydrous di- and monobasic phosphate were acquired from Synth ${ }^{\circledR}$ (Brazil). All reagents were of analytical grade. Milli-Q ultrapure water was used for all experiments.

\section{Methods}

Synthesis of gold nanoparticles and BSA capped AuNP

\section{Resveratrol assisted AuNP synthesis}

Gold nanoparticles were synthesized using $2.5 \mathrm{mM}$ sodium tetrachloroaurate (III) dehydrate and $1.67 \mathrm{mM}$ Resveratrol in water. Resveratrol solution was homogenized for 20 minute prior to gold addition. The mixture was allowed to react in a sealed volumetric flask at $22{ }^{\circ} \mathrm{C}$ for 12 hours under magnetic stirring.

\section{BSA capping}

BSA was added to the AuNP solution on ice bath to reach $5 \mathrm{mg}$ $\mathrm{mL}^{-1} \mathrm{BSA}$. The glass vials were hermetically sealed and the mixture was allowed to rest overnight at $4^{\circ} \mathrm{C} \pm 2$.

\section{BSA crosslinking onto AuNP}

BSA capped AuNP was exposed to $\gamma$-irradiation using ${ }^{60} \mathrm{Co}$ as radioactive source in Gammacell 220 irradiator (Atomic Energy of Canada Limited, Canada) at $0 \pm 1^{\circ} \mathrm{C}$ at the doses of $2.5,5,7.5,10$ and $15 \mathrm{kGy}$ and dose rate of $1.03 \mathrm{kGyh}^{-1}$, as determined Harwell Type by alanine dosimetry [14]. The samples were stored at refrigerated 4 $\pm 2^{\circ} \mathrm{C}$ prior to analysis.

\section{Nanoparticle characterization}

\section{Nanoparticle size}

Nanoparticle size was determined by Dynamic Light Scattering on a ZS 90 (Malvern, USA) device at scattering angle of $173^{\circ}$ degre- 
es. Results were reported by an average hydrodynamic diameter obtained by number using 3 sets of 3 runs of 120 seconds.

\section{Surface plasmon ressonance}

Samples were evaluated in spectrophotometer in the visible range, in order to asses particle properties.

\section{Particle morphology}

Protein Nanoparticle size and dimensions were determined by Transmission Electron Microscopy (NS-TEM) in a 120 kV JEM 1400 Plus electron microscope (Jeol, Japan) on ultrathin carbon film 400-mesh Cu grids (Ted Pella. Inc, USA) The samples (10 ul) were then transferred to the grids for 1 minute and specimen preparation was performed at room temperature.

\section{Protein crosslinking}

Bityrosine emission was scanned at $\lambda_{\mathrm{Em}}=350-600 \mathrm{~nm}$ using an excitation of $\lambda_{\text {Em }}=325 \mathrm{~nm}$ at a scan speed of $240 \mathrm{~nm} / \mathrm{min}$ on a Spectramax i3 (Molecular Devices, USA) and the values were normalized by taken native BSA spectrum as reference $[12,15]$.

\section{Results and Discussion}

\section{Resveratrol-mediated Synthesis of AuNP}

The use of resveratrol led to the formation of gold nanoparticles (AuNPs) of around $38.8 \pm 11.7 \mathrm{~nm}$ (hydrodynamic diameter) as determined by dynamic light scattering (DLS), with a surface plasmon resonance (SPR) peak at $530 \mathrm{~nm}$ for the undiluted sample at $25{ }^{\circ} \mathrm{C}$. The AuNPs produced were of spherical shape, and the SRP changing upon dilution as standard AuNPs, as revealed in figure 1 . These results corroborate literature concerning the resveratrol-mediated synthesis of AuNP. Mohanty., et al. [16] synthesized gold nanoparticle using resveratrol of spherical shape with $35 \mathrm{~nm}$, surface plasmon resonance band at $537 \mathrm{~nm}$ and zeta potential of $-21,2$ $\mathrm{mV}$. Sana., et al. [8] reported the green synthesis of gold nanoparticles using resveratrol show surface plasmon resonance band 540 $\mathrm{nm}$ and diameter of $14.55 \pm 2,20$ as estimated by TEM.

If compared to conventional routes such as the use of sodium citrate [17], the use of resveratrol leads to a different particle morphology, especially in terms of hydrodynamic size, considering that along with the reduction, resveratrol molecules are expected to cover gold nanoparticle surface, with among other features, con-

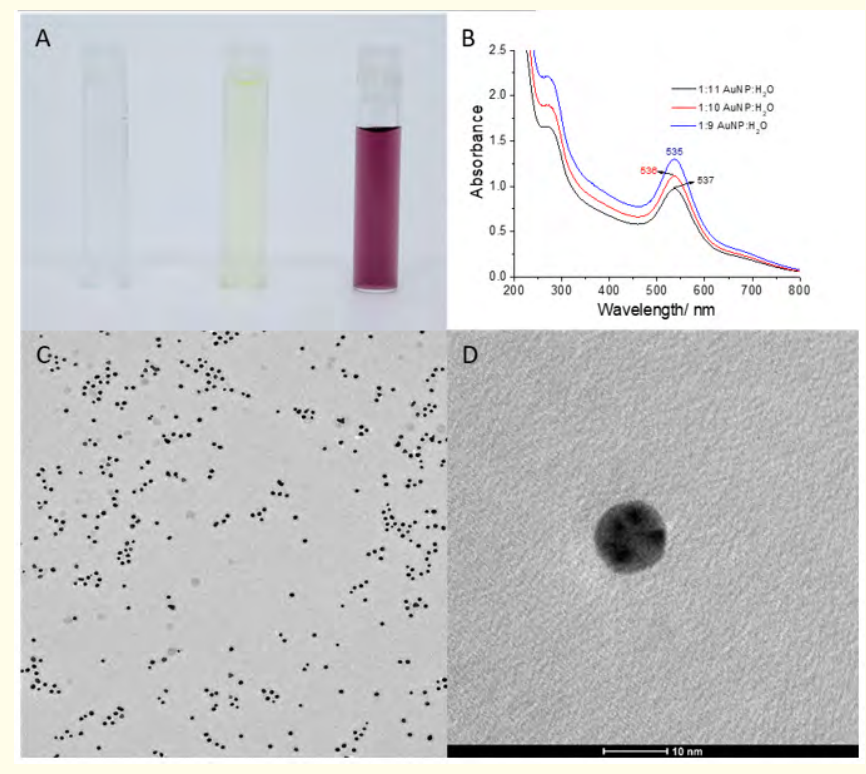

Figure 1: Gold nanoparticles produced via green synthesis using resveratrol. (a) Visual aspect of the samples before and after synthesis; (b) Surface plasmon resonance peak at $25 \mathrm{oC}$;

(c) Transmission electron microscopy images of the nanoparticles produced.

fer surface functionalization and stabilization of the nanoparticle $[8,15]$.

Mohanty [16] described that the mechanism of formation of resveratrol assisted gold nanoparticle relies on the reduction of $\mathrm{Au}^{3+}$ to $\mathrm{Au}^{0}$ by the antioxidant activity of resveratrol, which hypothesized to be similar to the reduction of $\mathrm{Fe}^{3+}$ to $\mathrm{Fe}^{2+}$ by other polyphenols with similar antioxidant activity [18].

\section{BSA capping onto AuNP}

The addition of BSA onto gold nanoparticles synthesized lead to an increase in the nanoparticle hydrodynamic size, with dimensions of $72.8 \pm 32.9 \mathrm{~nm}$ as determined by DLS. Also, SPR suffered a blue shift of $4 \mathrm{~nm}$, corresponding to $534 \mathrm{~nm}$, whereas undiluted AuNP presented $530 \mathrm{~nm}$ (Figure 2). This result provides an experimental evidence that BSA has been attached to the gold nanoparticle. A similar shift was observed in different works reporting binding of BSA onto gold nanoparticles by different methodologies [19]. 


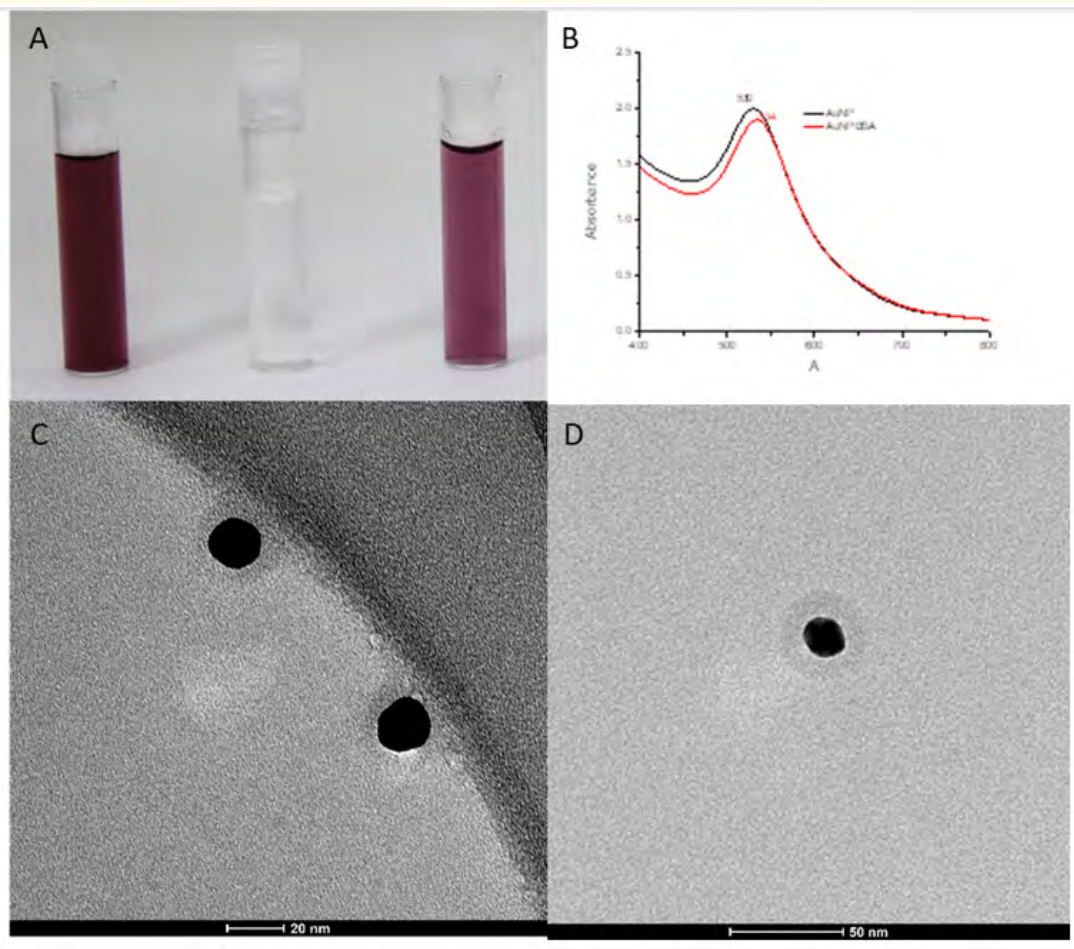

Figure 2: BSA capped AuNP produced via green synthesis using resveratrol. (a) Visual aspect and (b) Surface plasmon resonance peak at $25 \mathrm{oC}$ of the samples before and after

capping; (c) Transmission electron microscopy images of the BSA capped nanoparticles.

As there are no chemical ligands been added to the system, the spontaneous binding is more likely to occur via physical adsorption, as observed in the literature [20]. It is relevant to highlight that the change in the SPR without a notable change in the shape of the UV-Vis spectra reveals the maintenance of the properties of the pristine AuNP.

Corroborating the SPR and the DLS results, the micrographs (Figure $2 \mathrm{C}$ and $\mathrm{D}$ ) confirmed the albumin capping, identified on the surface surrounding the AuNPs end evidenced that the gold core remained preserved after capping.

The effect of gamma irradiation over AuNP and BSA capped AuNP

Irradiation has been applied to biomaterials over decades whether to promote crosslinking of the system or as a technique to sterilize and assure safety usage of biomedical devices. The effects of the exposition to irradiation, on the other hand, may lead to changes in nanoparticle characteristics.
The effects of irradiation over the visible spectra of the produced nanoparticles corresponded to a slightly red or blue-shift as a function of irradiation dose figure 3. In terms of Particle size, the effect of irradiation dose over AuNP (Figure $3 \mathrm{~A}$ ). In presence of BSA particle size shifted as a function of dose (figure $3 \mathrm{~B}$ ).
A

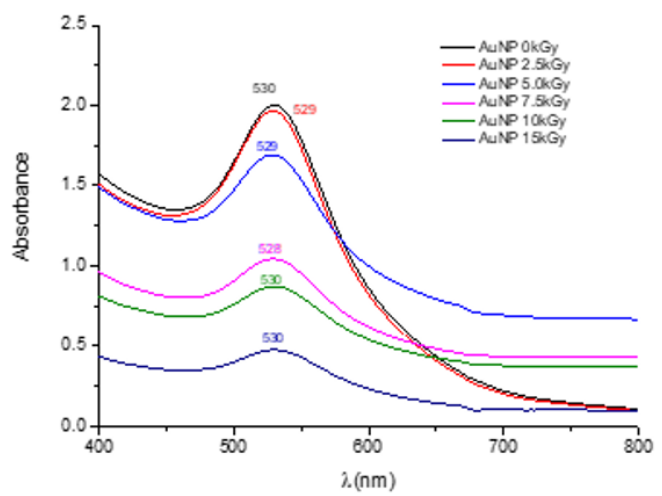


B
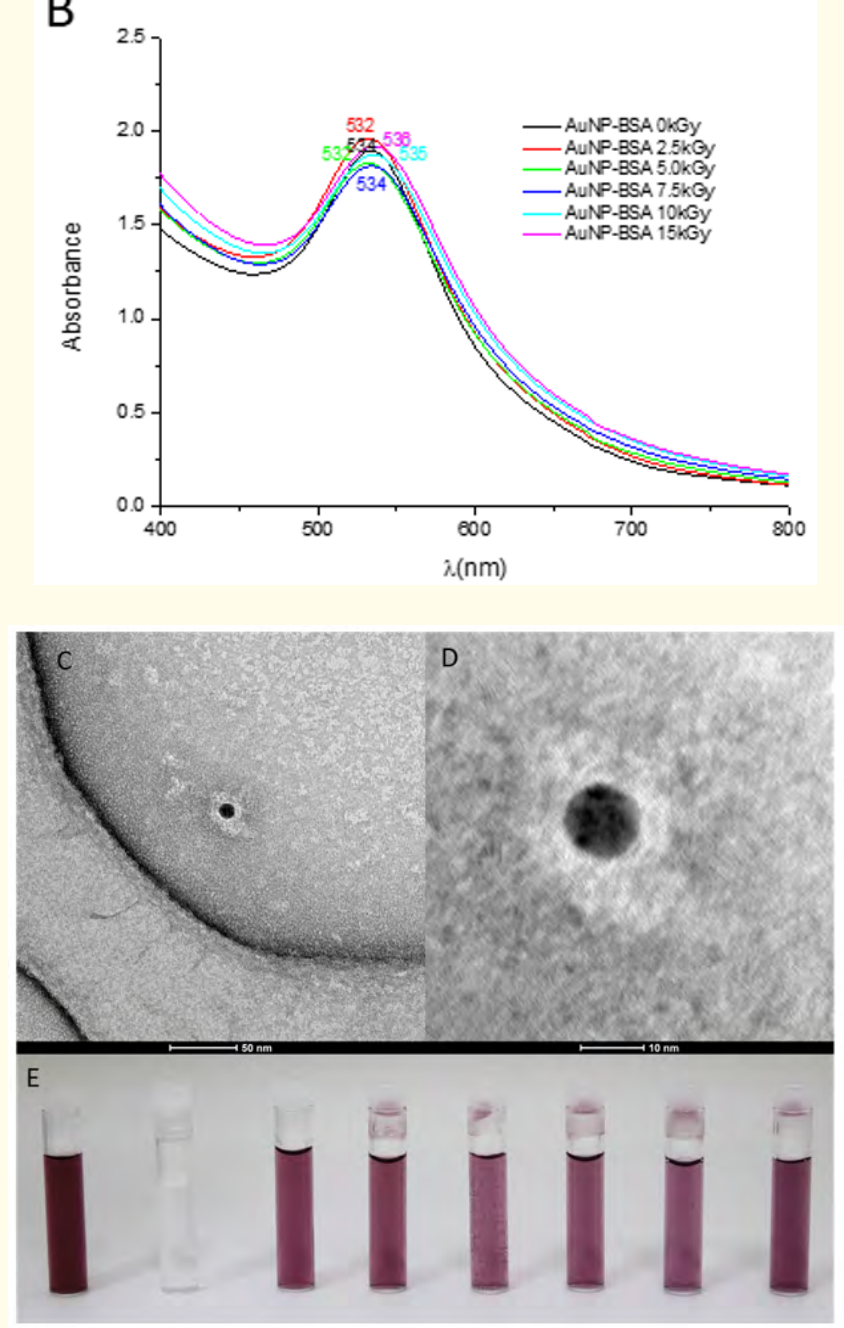

Figure 3: Visible spectra of AuNPS as function of radiation dose.

In terms of hydrodynamic radius, the effects of irradiation lead to a random profile, where particle size increase was registered at $2.5 \mathrm{kGy}$ and $7.5 \mathrm{kGy}$, whereas at 5 and $10 \mathrm{kGy}$, gold nanoparticles tended to a decrease in the hydrodynamic radius. Although such effects vary upon irradiation dose, the selection of the exact dose for sterilization of the produced systems depends on the microbial load of the sample prior to irradiation. Asnag., et al. [21] studies blend (PEO/starch) films doped with different concentration of gold nanoparticles demonstrated that gamma irradiation causes the structural variations in polymer blend chains on the embedding of AuNPs.

\section{BSA crosslinking onto AuNP}

As an attempt to understand the damage upon radiation exposition, we have evaluated the formation of bityrosine, a by-product resultant from the exposition of proteins to ionizing radiation, often present and a marker or intra- or intermolecular crosslinking.

Protein crosslinking levels of the BSA layer around the gold nanoparticles were assessed by means of bityrosine formation. The use of fluorescence measurement for the quantification of bityrosine is adequate based on the high fluorometric quantum yield of bityrosines associated to the high specificity of the fluorescence technique [22]. In terms of protein crosslinking induced by irradiation, fluorescence methods have been successfully applied for bityrosine monitoring in BSA $[12,15]$ and papain nanoparticles.

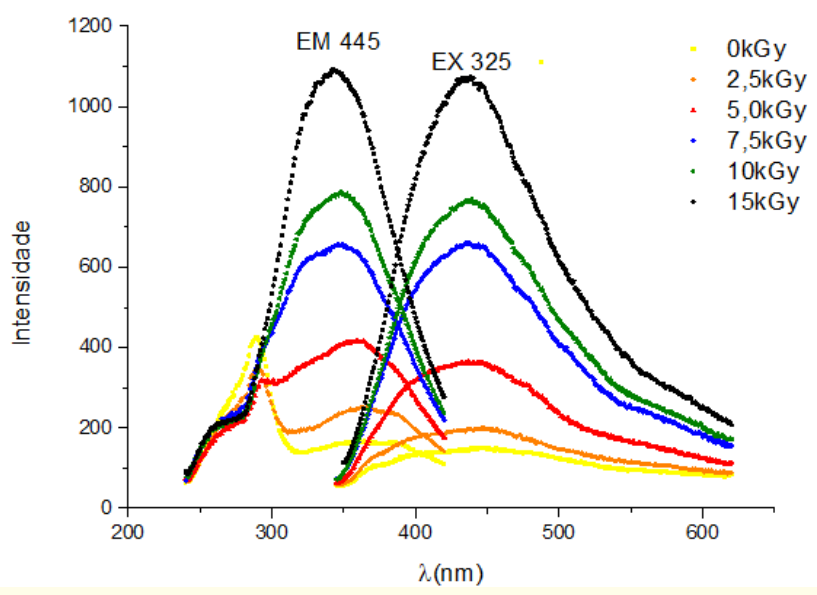

Figure 4: Fluorescence evaluation of the bityrosine linkages formed as a function of radiation dose (2.5 -10 kGy) in BSA capped AuNPs.

If compared to BSA crosslinked nanoparticles, the bytirosine formation is less intense [12,15], probably due to a distinct rearrangement of the BSA molecules as a result of the interaction with gold nanoparticles. These results suggest that the albumin layer formed surrounding the AuNP is crosslinked.

\section{Conclusions}

The method allowed the formation of AuNPs of spherical shape via green synthesis using resveratrol as a phytochemical. Apart from the lack of toxic compounds for the synthesis, the particles presented adequate size, size distribution and reproducibility as revealed by the assays performed. 
The BSA capping onto AuNPs was successfully achieved and experimentally demonstrated by the blue shift in the surface plasmon resonance peak without any the change in terms of the shape of the UV-vis spectra. Particle size increase of around $30 \mathrm{~nm}$ as a function of the BSA capping, as revealed by DLS, and the transmission electron microscopies provided a visual evidence of the capping onto the gold surface and its surroundings.

The effect of radiation dose in the given range of 2.5-10 kGy over AuNPS lead to destabilization and intense size changes as a function of dose, also linked to some induced agglomerations as revealed by the changes in the UV-Vis spectra. Upon BSA capping, such changes are negligible as a function of dose and which reinforces the beneficial effects of the capping in contrast to naked AuNPs, especially concerning the exposition to radiation, in which the gold core is preserved. This is particularly relevant especially if the particles are meant to be used for biological or biomedical applications in which the nanoparticle may be exposed to unusual environments and extreme conditions and must tolerate them.

Upon exposition to radiation, the formation of bityrosine was confirmed and identified to increase as radiation dose increased. Corroborating this information, the particle size increase (established around $\mathrm{xx} \mathrm{nm}$ ), also dose-dependent, evidenced the formation of a crosslinked BSA capping. However, the nature of such crosslinks and whether the signal comes from the BSA molecules in the capping or results from the isolated BSA present in the solution remain as a topic to be explored. At last, although our results evidenced a very effective capping onto gold nanoparticles, our revealed that some optimized conditions for synthesis, such as a reduction of albumin concentration and a possible purification step are further required for a direct application and optimization of the system.

\section{Acknowledgments}

The authors would like to thank Prof Dr. Newton Laboratório de Biofísica (IF-USP, São Paulo, Brazil), Centro de Biotecnologia (IPEN/ CNEN-SP), Fundação de Amparo à Pesquisa do Estado de São Paulo (FAPESP Project number 2015/13979-0), Conselho Nacional de Desenvolvimento Científico e Tecnológico (CNPq project number 402887/2013-1) and the Nuclear and Atomic Energy Agency (CRP code F22064) - IAEA for financial support.

\section{Bibliography}

1. Reddy GAK., et al. „Nano silver - a review”. International Journal of Advances in Pharmaceutical Sciences 2.1 (2012): 09.

2. Makarov VV., et al. "'Green" Nanotechnologies: Synthesis of Metal Nanoparticles Using Plants". Acta Naturae 6.1 (2014): 35-44.

3. Shukla r., et al. "Soybeans as a Phytochemical Reservoir for the Production and Stabilization of Biocompatible Gold Nanoparticles". Small 4.9 (2008): 1425-1436.

4. Viator JA., et al. "Gold Nanoparticle Mediated Detection of Prostate Cancer Cells Using Photoacoustic Flowmetry with Optical Reflectance". Journal of Biomedical Nanotechnology 6 (2010): 187-191.

5. Kattumuri V., et al. "Gum arabic as a phytochemical construct for the stabilization of gold nanoparticles: In vivo pharmacokinetics and X-ray-contrast-imaging Studies". Small 3 (2007): 333-341.

6. Kim YS., et al. "Suppressing effect of resveratrol on the migration and invasion of human metastatic lung and cervical cancer cells". Molecular Biology Reports 39 (2012): 8709-8716.

7. Chakraborty A., et al. "In vitro evaluation of the cytotoxic, anti-proliferative and anti-oxidant properties of pterostilbene isolated from Pterocarpus marsupium". Toxicology In Vitro 24 (2010): 1215-1228.

8. Sanna V., et al. "Single-step green synthesis and characterization of gold-conjugated polyphenol nanoparticles with antioxidant and biological activities". International Journal of Nanomedicine 9 (2014): 4935-4951.

9. Thipe VC., et al. „Development of resveratrol-conjugated gold nanoparticles: interrelationship of increased resveratrol corona on anti-tumor efficacy against breast, pancreatic and prostate cancers". International Journal of Nanomedicine 14 (2019): 4413-4428.

10. Cañaveras F., et al. "Role of the Functionalization of the gold nanoparticle surface on the formation of bioconjugates with human serum albumin". The Journal of Physical Chemistry C 116 (2012): 10430-10437. 
11. Karen Bolaños., et al. "Capping gold nanoparticles with albumin to improve their biomedical properties". International Journal of Nanomedicine 14 (2019): 6387-6406.

12. Queiroz RG., et al. "Radiation-synthesized protein-based drug carriers: Size-controlled BSA nanoparticles". International Journal of Biological Macromolecules 85 (2016): 82-91.

13. Achilli E., et al. "Preparation of protein nanoparticle by dynamic aggregation and ionizing-induced crosslinking". Colloids and Surfaces A: Physicochemical and Engineering Aspects 486 (2015): 161-171.

14. ISO/ASTM516-13 Standard Practice for Use of the AlanineEPR Dosimetry System.

15. Varca GHC., et al. "Irradiation as an alternative route for protein crosslinking: Cosolvent free BSA nanoparticles". Radiation Physics and Chemistry 24 (2016): 111-115.

16. Mohanty R K., et al. "Resveratrol stabilized gold nanoparticles enable surface loading ofdoxorubicin and anticancer activity". Colloids and Surfaces B: Biointerfaces 114 (2014): 138-143.

17. Zhao P., et al. "State of the art in gold nanoparticle synthesis". Coordination Chemistry Reviews 257.3-4 (2013): 638-665.

18. Perron NR., et al. "A review of the antioxidant mechanisms of polyphenol compounds related to iron binding". Cell Biochemistry and Biophysics 53 (2009): 75-100.

19. Cardoso-Avila P E., et al. "Gold nanoparticles surface modification using BSA and Cysteine". Proceedings of SPIE 8011 (2011): 801192-801197.

20. Mariam J., et al. "Albumin corona on nanoparticles - a strategic approach in drug delivery". Drug Delivery (2015): 1-9.

21. Asnag G M., et al. "Effect of gamma-irradiation on the structural, optical and electrical properties of PEO/starch blend containing different concentrations of gold nanoparticles". Radiation Effects and Defects in Solids 174.7-8 (2019): 579-595.

22. DiMarco T and iulivi C. „Current analytical method for the detection of dityrosine, a biomarker of oxidative stress in biological systems". Mass Spectrometry Reviews 26 (2006): 108-120.

\section{Assets from publication with us}

- Prompt Acknowledgement after receiving the article

- Thorough Double blinded peer review

- Rapid Publication

- Issue of Publication Certificate

- High visibility of your Published work

Website: www.actascientific.com/

Submit Article: www.actascientific.com/submission.php Email us: editor@actascientific.com

Contact us: +919182824667 